\title{
Intradialytic Modified Tai Chi Exercise among End-Stage Renal Disease Patients Undergoing Hemodialysis: An Exploratory Pilot Study
}

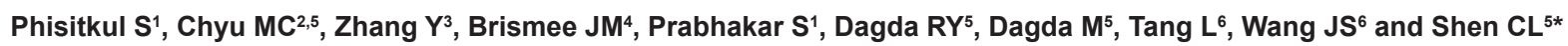

${ }^{1}$ Division of Nephrology, Department of Internal Medicine, Texas Tech University Health Sciences Center, Lubbock, TX, USA

${ }^{2}$ Graduate Healthcare Engineering Program, Whitacre College of Engineering, Texas Tech University, Lubbock, TX, USA

${ }^{3}$ Department of Community and Family Medicine, Texas Tech University Health Sciences Center, Lubbock, TX, USA

${ }^{4}$ Department of Rehabilitation Sciences, Texas Tech University Health Sciences Center, Lubbock, TX, USA

${ }^{5}$ Department of Pathology, Texas Tech University Health Sciences Center, Lubbock, TX, USA

${ }^{6}$ Department of Environmental Health Science, University of Georgia, Athens, Georgia, 30602, USA

\begin{abstract}
Background: Evidence suggests that intradialytic exercise benefits end-stage renal disease (ESRD) patients. We examined the feasibility of intradialytic modified Tai Chi (IMTC) among ESRD undergoing hemodialysis and assessed IMTC's impact on risk of falls, bone metabolism, oxidative stress damage, and quality of life in ESRD patients.

Methods: Forty-five ESRD patients were randomized to either non-exercising control group (CON, n=25) or IMTC group $(n=20)$ who performed IMTC exercise 3/week, $45 \mathrm{~min} /$ session while sitting in a recliner with one arm connected to the dialysis machine for 12 weeks. At baseline and 12 weeks, we measured risk-of-falls related parameters (balance, gait, and functioning strength), biomarkers of bone metabolism and oxidative stress damage, and quality of life.

Results: Thirty-four participants completed the study. The feedback from participants suggests that IMTC exercise is easy to learn and feasible. After 12 weeks, IMTC tended to lower tartrate- resistant acid phosphatase (bone resorption biomarker) and improved quality of life (role physical and vitality) compared to CON. There was no significant difference in balance, gait, and functional strength, possibly due to a ceiling effect. There was no significant difference in serum bone-specific alkaline phosphatase, calcium, phosphorus and intact parathyroid hormone levels, plasma 8-hydroxy-2'-deoxyguanosine levels, and other domains of quality of life. The CON group experienced a $20 \%$ decrease in 25 -hydroxy vitamin $D$ at the end of study.

Conclusions: IMTC exercise was safe and well accepted by ESRD patients. It improved quality of life, tended to suppress bone resorption, and helped maintain 25-hydroxy vitamin D levels in ESRD patients. These findings need to be confirmed in a larger, more sufficiently powered efficacy study.
\end{abstract}

Keywords: Intradialytic mind-body exercise; Hemodialysis; Bone metabolism; Quality of life; Risk of fall

\section{Summary}

Evidence suggests that intradialytic exercise benefits end-stage renal disease (ESRD) patients. Intradialytic modified Tai Chi (IMTC) exercise was safe and well accepted by ESRD patients. Relative to the non-exercising control group, IMTC improved quality of life, tended to suppress bone resorption, and helped maintain 25-hydroxy vitamin $\mathrm{D}$ levels in ESRD patients. However, there is no significant difference in risk-of-fall related parameters, including gait, balance, and functional strength, possibly due to a ceiling effect.

\section{Introduction}

End-stage renal disease (ESRD) patients generally suffer from lower physical activities, muscle performance, and quality of life (QOL) compared to healthy humans. Numerous studies have suggested that exercise-based rehabilitation programs could improve physical functioning and QOL in ESRD patients [1,2]. Exercise interventions during hemodialysis sessions while patients are connected to machine, i.e., intradialytic exercises, have become more and more popular as they are superior to interdialytic exercise programs (between two hemodialysis sessions when patients are not connected to machine) in terms of better adherence [3-9]. Intradialytic exercises have been shown to be safe [3], and can be safely performed in the first 2 hours of dialysis without cardiac decompensation [2]. A recent review summarizes the potential health benefits of intradialytic exercise programs for hemodialysis patients [2]. However, it is difficult to incorporate intradialytic exercise (e.g., cycle ergometer, bicycle training, resistance exercise) due to requirement of additional equipment, space, and personnel besides those for routine hemodialysis [2]. The present study proposes an intradialytic modified Tai Chi (IMTC) exercise that not only can be performed while the patient is sitting in a recliner with one arm connected to the hemodialysis machine, but also requires no exercise equipment (thus safer to practice), and is easy to learn. This intradialytic exercise has never been studied anywhere. Bone fractures are common in ESRD patients, resulting in a higher prevalence of vertebral and hip fractures than the general population [10,11]. Higher incidence rate of fracture in hemodialysis patients is likely due to higher risk of falls and renal osteodystrophy [12]. Because falls occur

*Corresponding author: Chwan-Li Shen, BB 198, $36014^{\text {th }}$ street, Department of Pathology, Texas Tech University Health Sciences Center, Lubbock, Texas 79430 9097, USA, E-mail: leslie.shen@ttuhsc.edu

Received May 15 2013; Accepted June 11, 2013; Published June 14, 2013

Citation: Phisitkul S, Chyu MC, Zhang Y, Brismee JM, Prabhakar S, et al. (2013) Intradialytic Modified Tai Chi Exercise among End-Stage Renal Disease Patients Undergoing Hemodialysis: An Exploratory Pilot Study. Altern Integ Med 2: 123. doi:10.4172/2327-5162.1000123

Copyright: @ 2013 Phisitkul S, et al. This is an open-access article distributed under the terms of the Creative Commons Attribution License, which permits unrestricted use, distribution, and reproduction in any medium, provided the original author and source are credited. 
Citation: Phisitkul S, Chyu MC, Zhang Y, Brismee JM, Prabhakar S, et al. (2013) Intradialytic Modified Tai Chi Exercise among End-Stage Renal Disease Patients Undergoing Hemodialysis: An Exploratory Pilot Study. Altern Integ Med 2: 123. doi:10.4172/2327-5162.1000123

Page 2 of 7

frequently in the hemodialysis population, simple fall prevention strategy may effectively reduce the risk of fracture in this population [13]. Among different forms of exercise, TC has been proven effective in reducing risk of falls in the elderly by improving gait [14-16], posture control and balance $[17,18]$. In ESRD patients, home-based TC improved functional mobility and muscle flexibility [19]. However, effects of IMTC on risk of fall in ESRD patients have never been investigated. In terms of renal osteodystrophy, adynamic bone disease is the most common type of renal osteodystrophy in hemodialysis patients, in which bone turnover is low due to excessive suppression of the parathyroid glands and aluminum deposition [20,21]. Management of hip fractures in ESRD patients is complicated by the heterogeneous nature of renal osteodystrophy and the inadequate methods of diagnosis currently available. Published studies have shown that exercise benefits bone metabolism [22,23]. However, very few studies have been reported on the effects of any form of exercise, let alone TC or IMTC, on bone metabolism in ESRD patients. An increased oxidative stress and decreased antioxidant capacity were associated with declined renal function in renal insufficiency and dialysis patients [24,25]. Strategies to reduce the oxidative stress damage in ESRD patients would be a reasonable approach to improve their health. Our recent study showed that a 6-month TC group exercise program significantly reduced urinary 8-hydroxy-2'-deoxyguanosine (8-OHdG, oxidative stress biomarker) in postmenopausal women, compared to those not practicing TC [26]. It is not known if IMTC exercise would reduce oxidative stress in ESRD patients. Comorbid medical conditions are common in ESRD patients, and are an importantcontributing factor to clinical outcomes and QOL [27]. Intradialytic aerobic and resistance exercises benefit health-related QOL in ESRD patients [2]. On the other hand, TC has been shown to improve QOL in study subjects with various health conditions [2830]. However, no study has been reported on IMTC's effects on QOL in ESRD patients. In light of the above discussion, the specific aims of this exploratory pilot study were (i) to examine the feasibility of IMTC among ESRD, and (ii) to assess IMTC's impact on risk of falls, bone metabolism, oxidative stress damage and QOL in study participants. We hypothesized that it would be feasible to teach study participants IMTC exercise and that IMTC would reduce risk of falls, improve bone metabolism and QOL, and decrease oxidative stress in ESRD subjects.

\section{Methods}

\section{Design}

This was a 12-week, randomized-controlled trial to investigate the feasibility of IMTC among ESRD undergoing hemodialysis, and to assess IMTC's impact on risk of falls, bone metabolism, oxidative stress damage, and quality of life in ESRD patients. Outcome data were collected at the baseline and the end of the 12-week study. The outcome assessors were blinded to the participant's treatment assignment. This study was approved by the local Institutional Review Board.

\section{Participants}

Participants were recruited from two local dialysis units in West Texas, USA. Informed consents were obtained from all participants. In addition, we also obtained patient consent for the use of their images for publication. Inclusion criteria included 18 to 80 years of age, adequate dialysis delivery with single pool Kt/V $\geq 1.4$ in last 3 months, and good compliance with dialysis treatment. Exclusion criteria included being on dialysis for less than three months, patients in a catabolic state, active intravenous drug use, vascular access for hemodialysis at lower extremity, contraindications to IMTC, and unable to give informed consent. All participating subjects were asked to maintain their regular diet, medication if any, normal daily activities and lifestyle throughout the study. Following recruitment and screening, 45 eligible subjects were randomly assigned (stratified by age and gender) to one of two groups: (i) IMTC ( $\mathrm{n}=20$, excluding 4 withdrew immediately after randomization) and (ii) control $(\mathrm{CON}, \mathrm{n}=25)$.

\section{Intervention}

Patients in the IMTC group practiced 45 minutes of IMTC during the first 2 hours of each 136 hemodialysis session, 3/week for 12 weeks, while receiving usual care. Each 45-minute exercise session consisted of 5 minutes of warm-up exercise, 35 minutes of IMTC, and 5 minutes of cool-down exercise. The warm-up exercise was designed to loosen major joints for the subsequent IMTC, along with controlled breathing.

The current IMTC exercise was based on the popular 24-form Tai Chi [31] with a difference that IMTC did not involve any upper body movements or body translation by foot, so that a participant could perform the exercise while sitting in a recliner with one arm held still that had needle access for hemodialysis. The exercise features performing the arm movements of the popular 24-form Tai Chi with legs. Figure 1 illustrates how some of those movements were adapted for IMTC. The traditional TC's unique ingredients, viz. physical relaxation, mental concentration, and deep breathing, were still intact and emphasized in the practice of IMTC. The cool-down exercise gradually brought the body condition down to its pre-exercise level. An instructor taught each IMTC participant individually. All IMTC participants were able to learn the exercise in 3 sessions. Thereafter, patients were able to perform IMTC by themselves following a handout and under the supervision of the instructor during each session. Before and after each IMTC session, study participants were monitored about the occurrence of any side effects in association with IMTC. The compliance and the length of practice time for each participant's IMTC session were recorded. Participants in the CON were asked to continue their regular activities throughout the study period, while receiving usual care.

\section{Measurement of balance, gait, and functional strength}

Static and dynamic balance were assessed by Berg Balance Test, consisting of 14 different tasks related to balance and postural control scored from 0 (inability) to 4 (ability) to perform the required tasks [3234]. Gait, balance and fall risk were assessed by Dynamic Gait Index which evaluates usual steady-state walking and walking during more challenging tasks. The test consists of eight gait tasks with a grading system from 0 to 3 and a maximum score of 24 [35,36]. Functional strength was assessed by the Timed "Up and Go" test [37].

\section{Biomarker measurement}

The data of calcium (Ca), phosphorus $(\mathrm{P})$ and $\mathrm{Ca} \times \mathrm{P}$ product routinely collected at dialysis clinics as part of dialysis management were used in the present study. Overnight fasting blood samples were collected, processed for serum and plasma samples, and stored at $-8^{\circ} \mathrm{C}$ for later analyses. Serum BAP and tartrate-resistant acid phosphatase (TRAP) (Quidel Corporation, San Diego, CA) and 1,25-dihydroxy vitamin D (1,25-OH-VitD) (Immunodiagnostics System, Fountain Hills, AZ) levels were measured by the respective kits. Serum intact parathyroid 9 hormone (iPTH) and 25-hydroxy vitamin D (25-OHVitD) were measured by Quest Diagnostics (Irving, TX). Plasma 8-OHdG level was determined accordingly [38-40].

\section{Quality of life}

General health status was measured with the Medical Outcomes Study Short Form Health Survey (SF-36, v2) [41-43]. The SF-36 consists 
Citation: Phisitkul S, Chyu MC, Zhang Y, Brismee JM, Prabhakar S, et al. (2013) Intradialytic Modified Tai Chi Exercise among End-Stage Renal Disease Patients Undergoing Hemodialysis: An Exploratory Pilot Study. Altern Integ Med 2: 123. doi:10.4172/2327-5162.1000123

Page 3 of 7

(a) "Commencing".

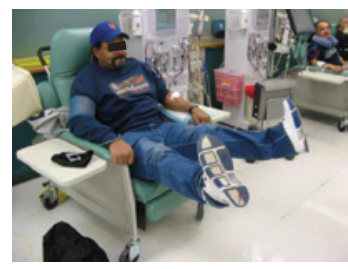

(d) "Repulse monkey".

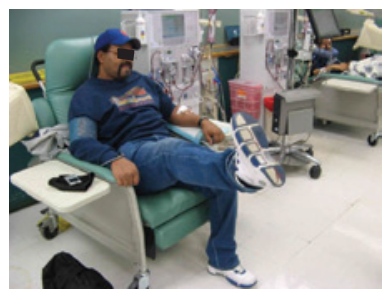

(g) "Cloud hand".

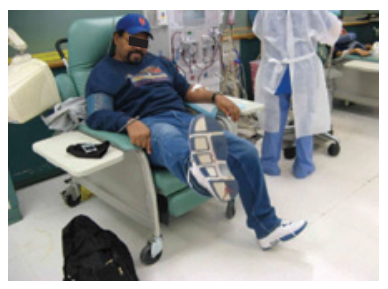

(b) "Part wild horse's mane".

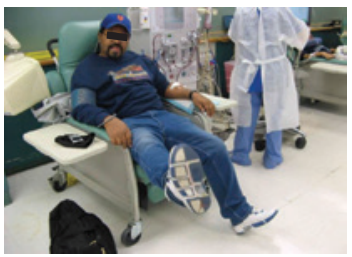

(e) "Grasp bird's tail - push".

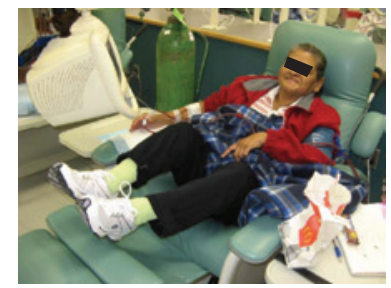

(c) "White crane spreads wings".

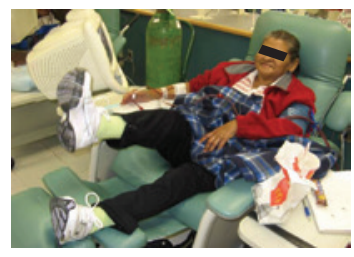

(f) "Single whip".

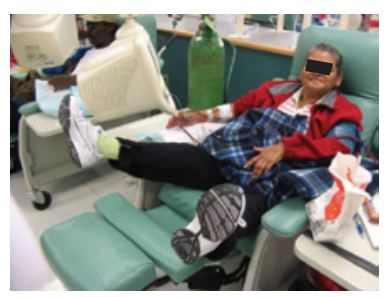

(h) "Left kick". Yellow arrowhead indicates the direction of motion.

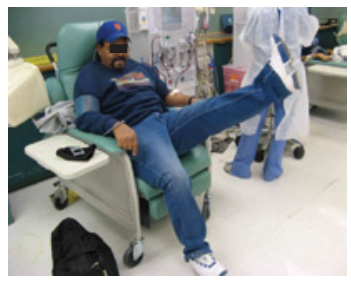

Figure 1: Examples of the intradialytic modified Tai Chi (IMTC) movements

of eight domains of health (physical function, bodily pain, general health, vitality, mental health, social function, and role of physical and emotional health) in the conduct of daily activities [41].

\section{Statistical analysis}

As the first study to explore IMTC's impact on ESRD, we used Cohen's effect size (ES) index to estimate the sample size [44]. A sample size of 50 subjects with an anticipated attrition rate of $20 \%$ over 12 weeks of intervention was determined to produce a final sample size of 40 subjects, and to provide $80 \%$ power to detect medium to large effect size at alpha level of 0.05 .

Statistical analysis was conducted using SPSS 16.0 (Chicago, IL, USA). All variables were expressed as means \pm standard deviations (SD) or standard error of the mean (SEM). Independent $t$-tests were conducted to compare the differences in outcomes between the IMTC and the CON groups at the baseline and the end point for preliminary bi-variate assessment. The change of each outcome was presented as percentage change relative to the baseline. The significance level was set at $p<0.05$. Changes of SF-36, balance, gait, and functional strength were assessed by independent t-tests, and significance levels were adjusted accordingly based on the number of items compared in each outcome measure. For instance, the significance level was $\mathrm{p}<0.05 / 8=0.006$ for SF-36. All biomarker outcome changes (from the baseline to 12 weeks) were analyzed using MANOVA procedure to determine differences between groups while Bonferroni adjustment was used for multiple comparisons. SPSS adjusts the actual $p$ value by applying the Bonferroni correction backwards so that the p-values of multiple comparisons can be compared directly to 0.05 .

\section{Results}

Among the 45 ESRD patients recruited (16 women and 29 men, 25 in CON and 20 in IMTC), six subjects (24\%) in the CON (final $n=19$ ) and five subjects $(25 \%)$ in the IMTC (final $n=15)$ withdrew due to death $(n=2)$, lost of interest $(n=8)$ and receiving kidney transplant $(n=1)$. Compliance for the IMTC was $89 \%$, while the average actual exercise time per session for the participants was $26 \mathrm{~min}$, i.e., $58 \%$ of the 45 min required. During the study period, no side effect that occurred in association with the intervention was reported by the subjects or observed by the research staff. There was no incidence of needle dislodgement due to IMCT during hemodialysis sessions, and IMTC did not interfere with the nurses' care of the hemodialysis patients.

At the baseline, the demographic and medical history characteristics were similar between CON and IMTC (Table 1). Because no statistically significant differences between the participants who withdrew from the study and those who completed the study were observed in any parameters, only the data of those who completed the study are presented. The MANOVA results showed that the 25-OH-vitamin $\mathrm{D}$ change is statistically significant $(\mathrm{F} 1,1=6.41, \mathrm{p}=0.02)$ between the exercise and the control group after the 12-week intervention. No significant findings were observed for other biomarkers. SPSS output 
Citation: Phisitkul S, Chyu MC, Zhang Y, Brismee JM, Prabhakar S, et al. (2013) Intradialytic Modified Tai Chi Exercise among End-Stage Renal Disease Patients Undergoing Hemodialysis: An Exploratory Pilot Study. Altern Integ Med 2: 123. doi:10.4172/2327-5162.1000123

Page 4 of 7

\begin{tabular}{|l|l|l|l|}
\hline Characteristic & CON $(\mathbf{n = 2 5})$ & IMTC $(\mathbf{n = 2 0 )}$ & P-value* \\
\hline Age (years), mean \pm SD & $57.4 \pm 11.7$ & $50.4 \pm 15.1$ & NS \\
\hline Sex & & & NS \\
\hline Male & $14(56 \%)$ & $9(45 \%)$ & \\
\hline Female & $11(44 \%)$ & $11(55 \%)$ & \\
\hline Weight $(\mathrm{kg})$, mean \pm SD & $82.2 \pm 32.4$ & $75.5 \pm 16.1$ & NS \\
\hline Height $(\mathrm{m})$, mean \pm SD & $1.66 \pm 0.1$ & $1.65 \pm 0.07$ & NS \\
\hline Body Mass Index (kg/m2), mean \pm SD & $30.1 \pm 10.9$ & $27.5 \pm 5.3$ & NS \\
\hline General health questions [n (\%)] & & & \\
\hline General health rated "good" & $11(44)$ & $7(35)$ & NS \\
\hline History of osteoarthritis & $7(28)$ & $5(25)$ & NS \\
\hline Severe joint or muscle pain & $11(44)$ & $8(40)$ & NS \\
\hline $\begin{array}{l}\text { Back or leg pain to prevent from } \\
\text { walking }\end{array}$ & $7(28)$ & $6(30)$ & NS \\
\hline Use of walker or cane for walking & $6(24)$ & $5(25)$ & NS \\
\hline History of diabetes & $12(48)$ & $14(70)$ & NS \\
\hline History of hypertension & $16(64)$ & $14(70)$ & NS \\
\hline Trouble sleeping & $14(56)$ & $10(50)$ & NS \\
\hline
\end{tabular}

NS, not statistical significance $(P>0.05)$

Table 1: Baseline characteristics of participants.

\begin{tabular}{|c|c|c|c|c|}
\hline Parameters & CON $(\mathrm{N}=19)$ & IMTC ( $\mathrm{N}=15)$ & t-test & $p$ value \\
\hline \multicolumn{5}{|l|}{ Berg balance test } \\
\hline Baseline (week 0) & $48.9 \pm 4.5$ & $49.1 \pm 9.0$ & -0.1 & 0.921 \\
\hline End (12 weeks) & $48.6 \pm 8.9$ & $51.1 \pm 5.5$ & -0.973 & 0.338 \\
\hline Changes (week 12-0) & $-0.8 \pm 1.6$ & $2.0 \pm 1.6$ & -1.225 & 0.23 \\
\hline \multicolumn{5}{|l|}{ Dynamic gait index } \\
\hline Baseline (week 0) & $22.2 \pm 1.1$ & $21.6 \pm 3.8$ & 0.561 & 0.579 \\
\hline End (12 weeks) & $20.5 \pm 5.6$ & $22.8 \pm 1.0$ & -1.615 & 0.119 \\
\hline Changes (week 12-0) & $-1.9 \pm 5.0$ & $1.1 \pm 3.8$ & -1.72 & 0.098 \\
\hline \multicolumn{5}{|c|}{ Timed up \& go (seconds) } \\
\hline Baseline (week 0) & $14.8 \pm 4.5$ & $14.4 \pm 8.2$ & 0.2 & 0.843 \\
\hline End (12 weeks) & $16.0 \pm 8.4$ & $12.9 \pm 2.8$ & 1.381 & 0.177 \\
\hline Changes (week 12-0) & $1.2 \pm 5.8$ & $-1.4 \pm 6.0$ & 1.286 & 0.208 \\
\hline
\end{tabular}

Data was presented as means $\pm \mathrm{SD}$

\#indicates a marginal difference of score change between the two groups $(0.05<p<0.1)$

Table 2: Comparison of balance, gait, and timed up and go results between the control and the exercise groups.

also showed that the observed power of MANOVA model ranged from 0.05 (8-OHdG) to 0.69 (25-OH-vitamin $\mathrm{D})$, which indicated our study was apparently under-powered. Therefore, we only report our bivariates analysis results in details below.

T-tests showed no statistical difference in risk-of-fall-related tests (the scores of Berg Balance Test, Dynamic Gait Index, or Timed Up \& Go test) between CON and IMTC either at baseline or at 12 weeks (Table 2). The results revealed a marginal difference of score changes in dynamic gait index with IMTC group showing an increased trend while CON group showing a decreased trend (Table 2, $\mathrm{t}=-1.720, \mathrm{p}=0.098$ ).

Data of serum and plasma biomarkers of the two groups are compared in Table 3. At the baseline, except for the 1,25-OH-VitD concentrations, there was no significant difference in all biomarkers between CON and IMTC. After 12 weeks, the following were observed: (i) Compared to CON, the IMTC exercise intervention did not change $\mathrm{BAP}, \mathrm{Ca}, \mathrm{P}, \mathrm{Ca} \times \mathrm{P}$, and $\mathrm{iPTH}$, but tended to suppress TRAP level ( $p=0.056$ ). (ii) The level of $1,25-\mathrm{OH}-\mathrm{VitD}$ in $\mathrm{CON}$ continued to be higher than IMTC $(\mathrm{p}=0.01)$. As for the changes, a $20 \%$ decrease in 25-OH-VitD level in CON was observed while IMTC did not show the similar trend $(\mathrm{t}=-1.911, \mathrm{p}=0.065)$ (Table 3$)$.

\begin{tabular}{|c|c|c|c|c|}
\hline Parameters & $\operatorname{CON}(\mathrm{N}=19)$ & IMTC (N=15) & t-test & $p$ value \\
\hline \multicolumn{5}{|l|}{ BAP (U/L) } \\
\hline Baseline (week 0) & $87.6 \pm 26.3$ & $59.3 \pm 14.5$ & 0.876 & 0.387 \\
\hline End (12 weeks) & $92.1 \pm 25.2$ & $59.5 \pm 20.4$ & 0.968 & 0.34 \\
\hline Changes (week 12-0) & $4.5 \pm 9.1$ & $0.2 \pm 7.4$ & 0.343 & 0.734 \\
\hline \multicolumn{5}{|l|}{ TRAP (U/L) } \\
\hline Baseline (week 0) & $9.9 \pm 1.7$ & $8.5 \pm 1.3$ & 0.576 & 0.572 \\
\hline End (12 weeks) & $10.7 \pm 1.8$ & $6.9 \pm 0.8^{\wedge \wedge}$ & 1.692 & 0.056 \\
\hline Changes (week 12-0) & $0.8 \pm 0.7$ & $-1.6 \pm 1.6$ & 1.466 & 0.152 \\
\hline \multicolumn{5}{|l|}{$\mathrm{Ca}(\mathrm{mg} / \mathrm{dL})$} \\
\hline Baseline (week 0) & $8.9 \pm 0.1$ & $9.0 \pm 0.1$ & 0.845 & 0.404 \\
\hline End (12 weeks) & $8.8 \pm 0.2$ & $8.6 \pm 0.3$ & 0.723 & 0.475 \\
\hline Changes (week 12-0) & $-0.1 \pm 0.1$ & $-0.4 \pm 0.2$ & 1.437 & 0.161 \\
\hline \multicolumn{5}{|l|}{$P(m g / d L)$} \\
\hline Baseline (week 0) & $5.5 \pm 0.5$ & $5.5 \pm 0.4$ & -0.0047 & 0.996 \\
\hline End (12 weeks) & $5.8 \pm 0.4$ & $6.4 \pm 0.5$ & -0.844 & 0.405 \\
\hline Changes (week 12-0) & $0.3 \pm 0.4$ & $0.9 \pm 0.6$ & -0.458 & 0.651 \\
\hline \multicolumn{5}{|l|}{$\mathrm{Ca} \times \mathrm{P}$} \\
\hline Baseline (week 0) & $48.8 \pm 4.1$ & $49.6 \pm 3.4$ & -0.127 & 0.9 \\
\hline End (12 weeks) & $51.5 \pm 4.1$ & $55.1 \pm 4.5$ & -0.572 & 0.572 \\
\hline Changes (week 12-0) & $2.6 \pm 3.6$ & $3.53 \pm 5.4$ & -0.136 & 0.892 \\
\hline \multicolumn{5}{|l|}{ iPTH (pg/mL) } \\
\hline Baseline (week 0) & $692.5 \pm 141.6$ & $545.3 \pm 150.3$ & 0.708 & 0.484 \\
\hline End (12 weeks) & $769.1 \pm 205.4$ & $365.9 \pm 78.5$ & 1.665 & 0.106 \\
\hline Changes (week 12-0) & $76.5 \pm 143.8$ & $-179.4 \pm 163.6$ & 1.176 & 0.248 \\
\hline \multicolumn{5}{|l|}{ 25-OH-vitamin D (ng/mL) } \\
\hline Baseline (week 0) & $26.0 \pm 2.5$ & $28.4 \pm 3.7$ & -0.536 & 0.596 \\
\hline End (12 weeks) & $20.4 \pm 2.6^{*}$ & $27.6 \pm 3.6$ & -1.65 & 0.109 \\
\hline Changes (week 12-0) & $5.6 \pm 2.1$ & $1.1 \pm 2.9 \#$ & -1.911 & 0.065 \\
\hline \multicolumn{5}{|l|}{ 1,25-OH-vitamin D (pmol/L) } \\
\hline Baseline (week 0) & $14.6 \pm 2.4$ & $4.8 \pm 0.4^{\wedge}$ & 3.45 & 0.002 \\
\hline End (12 weeks) & $13.6 \pm 2.6$ & $5.3 \pm 0.5^{\wedge}$ & 2.71 & 0.01 \\
\hline Changes (week 12-0) & $-1.0 \pm 1.0$ & $0.5 \pm 0.6$ & -1.081 & 0.288 \\
\hline \multicolumn{5}{|l|}{ 8-OHdG (ng/mL) } \\
\hline Baseline (week 0) & $0.324 \pm 0.048$ & $0.375 \pm 0.038$ & -0.799 & 0.431 \\
\hline End (12 weeks) & $0.329 \pm 0.053$ & $0.353 \pm 0.043$ & -0.335 & 0.74 \\
\hline Changes (week 12-0) & $0.005 \pm 0.026$ & $-0.022 \pm 0.030$ & 0.676 & 0.504 \\
\hline
\end{tabular}

Data was presented as means \pm SEM

BAP, bone-specific alkaline phosphatase; TRAP, tartrate-resistant acid phosphatase; $\mathrm{Ca}$, corrected calcium; $\mathrm{P}$, phosphate; $\mathrm{PTTH}$, intact parathyroid hormone; 8-OHdG, 8-hydroxydeoxyguanosine.

$\wedge$ indicates significant difference between the two groups at the same collection time $(p<0.05)$.

$\wedge \wedge$ indicates marginal difference between the two groups at the same collection time $(0.05<p<0.1)$

*indicates significant difference of change from the baseline ( 0 week) to the end of study (12 weeks) in the same group $(p<0.05$, paired t-test value and actual $p$-value not listed).

\#indicates marginal difference of score change between the two groups $(0.05<p<$ $0.1)$.

Table 3: Comparison of serum and plasma biomarkers results between the control and the modified groups.

Results for the Quality of Life (QOL) of the two groups are compared in Table 4 . At the baseline or at 12 weeks, there was no difference in any domain of QOL between CON and IMTC except for role physical ( $\mathrm{p}=0.034)$. After 12 weeks, IMTC significantly improved in physical function, role physical, and mental health, while CON showed no change in any domain (Table 4). The data also revealed significant difference of changes in scores of role physical $(t=-2.199, p=0.004)$ and vitality $(\mathrm{t}=-1.736, \mathrm{p}=0.009)$ between CON and IMTC groups. However, except for changes in role physical, the above t-test results were not statistically significant after Bonferroni adjustment $(\mathrm{p}>0.05 / 8=0.006)$. 
Citation: Phisitkul S, Chyu MC, Zhang Y, Brismee JM, Prabhakar S, et al. (2013) Intradialytic Modified Tai Chi Exercise among End-Stage Renal Disease Patients Undergoing Hemodialysis: An Exploratory Pilot Study. Altern Integ Med 2: 123. doi:10.4172/2327-5162.1000123

Page 5 of 7

\begin{tabular}{|c|c|c|c|c|}
\hline Parameters & $\operatorname{CON}(\mathrm{N}=19)$ & IMTC (N=15) & t-test & $p$ value \\
\hline \multicolumn{5}{|l|}{ Physical function } \\
\hline Baseline (week 0) & $44.7 \pm 23.9$ & $50.6 \pm 9.0$ & -0.575 & 0.569 \\
\hline End (12 weeks) & $46.6 \pm 28.8$ & $60.6 \pm 29.5^{*}$ & -1.374 & 0.179 \\
\hline Changes (week 12-0) & $1.9 \pm 17.7$ & $10.0 \pm 16.1$ & -1.603 & 0.119 \\
\hline \multicolumn{5}{|l|}{ Role physical } \\
\hline Baseline (week 0) & $67.7 \pm 22.6$ & $50.0 \pm 23.1^{\wedge}$ & 2.216 & 0.034 \\
\hline End (12 weeks) & $63.2 \pm 21.3$ & $64.5 \pm 25.7^{*}$ & -0.166 & 0.869 \\
\hline Changes (week 12-0) & $-5.2 \pm 29.7$ & $14.5 \pm 19.8 \#$ & -2.199 & 0.004 \\
\hline \multicolumn{5}{|l|}{ Bodily pain } \\
\hline Baseline (week 0) & $68.7 \pm 22.5$ & $56.2 \pm 19.2^{\wedge \wedge}$ & 1.704 & 0.098 \\
\hline End (12 weeks) & $63.2 \pm 21.3$ & $62.9 \pm 19.0$ & 0.0407 & 0.968 \\
\hline Changes (week 12-0) & $-5.5 \pm 26.8$ & $6.7 \pm 23.1$ & -1.392 & 0.174 \\
\hline \multicolumn{5}{|l|}{ General health } \\
\hline Baseline (week 0) & $51.1 \pm 28.0$ & $40.4 \pm 16.2$ & 1.313 & 0.199 \\
\hline End (12 weeks) & $50.3 \pm 23.8$ & $43.1 \pm 16.1$ & 0.975 & 0.337 \\
\hline Changes (week 12-0) & $-0.7 \pm 17.5$ & $2.3 \pm 12.0$ & -0.58 & 0.566 \\
\hline \multicolumn{5}{|l|}{ Vitality } \\
\hline Baseline (week 0) & $54.5 \pm 21.9$ & $49.5 \pm 14.6$ & 0.742 & 0.464 \\
\hline End (12 weeks) & $52.0 \pm 23.1$ & $57.9 \pm 18.2$ & -0.791 & 0.435 \\
\hline Changes (week 12-0) & $-2.4 \pm 17.3$ & $8.3 \pm 18.2 \#$ & -1.736 & 0.009 \\
\hline \multicolumn{5}{|l|}{ Social functioning } \\
\hline Baseline (week 0) & $67.3 \pm 25.7$ & $66.6 \pm 19.8$ & 0.0853 & 0.933 \\
\hline End (12 weeks) & $68.0 \pm 26.1$ & $70.8 \pm 26.5$ & -0.302 & 0.765 \\
\hline Changes (week 12-0) & $0.7 \pm 20.3$ & $4.1 \pm 29.7$ & -9.397 & 0.694 \\
\hline \multicolumn{5}{|l|}{ Role emotional } \\
\hline Baseline (week 0) & $71.7 \pm 32.2$ & $71.6 \pm 29.8$ & 0.0085 & 0.993 \\
\hline End (12 weeks) & $63.4 \pm 30.4$ & $75.0 \pm 21.5$ & -1.236 & 0.226 \\
\hline Changes (week 12-0) & $-8.3 \pm 30.6$ & $3.3 \pm 16.9$ & -1.315 & 0.198 \\
\hline \multicolumn{5}{|l|}{ Mental health } \\
\hline Baseline (week 0) & $72.8 \pm 20.5$ & $70.0 \pm 16.1$ & 0.425 & 0.673 \\
\hline End (12 weeks) & $73.0 \pm 23.0$ & $79.0 \pm 15.1^{*}$ & -0.857 & 0.398 \\
\hline Changes (week 12-0) & $0.2 \pm 21.3$ & $9.0 \pm 6.8$ & -1.517 & 0.139 \\
\hline
\end{tabular}

Data was presented as means $\pm \mathrm{SD}$.

$\wedge$ indicates significant difference between the two groups at the same collection time $(p<0.05)$.

$\wedge \wedge$ indicates a marginal difference between the two groups at the same collection time $(0.05<p<0.1)$

*indicates significant difference of change from the baseline ( 0 week) to the end of study ( 12 weeks) in the same group $(p<0.05$, paired t-test value and actual $p$-value not listed)

\#indicates significant difference of score change between the two groups $(p<0.05)$ Table 4: Comparison of Quality of Life results between the control and the exercise groups.

\section{Discussion}

This study was the first to evaluate the IMTC's impact in ESRD patients. The study suggests that IMTC may be an alternative exercise for ESRD patients based on the following results: (i) IMTC exercise is feasible, safe, and well accepted by ESRD patients, (ii) IMTC improves quality of life parameters, and (iii) IMTC maintains 25-OH-VitD levels in ESRD patients.

Physical activity deficiency is common among ESRD patients undergoing hemodialysis [45-48]. Physicians in general lack awareness of and ignore the importance of exercise for hemodialysis patients [8]. ESRD patients also tend to have a low interest in exercise due to fatigue, quick changes in medical status, functional dependency, depression, as well as lack of exercise equipment, regimen prescription, time, transportation, and motivation.

IMCT investigated in the current study may be an exercise program that can minimize those barriers. IMTC was taught to each

subject by one investigator, and following the first three sessions, subjects performed the IMTC independently under supervision of the investigator. Despite the difference between IMTC and the traditional TC, physical relaxation, mental concentration and deep breathing remained emphasized in the practice of IMTC and could be the reasons for the improvements observed in QOL. Because no negative side effects were recorded as a consequence of IMTC, we estimate that the intervention could be appropriately supervised by the nursing staff and would not require additional staffing. Future research evaluating the use of IMTC taught through video technology under supervision of nursing staff would be valuable.

The findings that no significant changes observed in balance, gait, and functional strength after 12 weeks of IMTC intervention may be due to a ceiling effect. High risk of fall is indicated by Berg Balance Test score $<36$, Dynamic Gait Index score $<19$, or Timed Up \& Go $>20$ seconds. In this study, except for one participant who was at risk of fall, all the others performed well in all three tests at the baseline, leaving little room for improvement in the follow-up assessment. The subject at risk of fall at the baseline improved in balance, gait, and functional strength after 12 weeks of IMTC intervention. Such result suggests an exclusion criterion of patients without risk of fall in future studies. In addition, the fact that our ESRD patients performed IMTC exercise in a seated position, instead of a standing position, could explain the lack of improvements in balance, gait, and functional strength. Practice of TC in a seated position involves minimal training of balance control compared to standing [49]. Future studies including weight bearing IMTC exercises would be valuable to determine their feasibility in this population during dialysis and if the inclusion of weight bearing exercises would positively affect the outcomes related to gait, balance and falls.

Dialysis patients have a higher risk of fall post-hemodialysis than pre-hemodialysis, and most of the falls occur post-hemodialysis. After 4 hours of dialysis and 3-5 $\mathrm{kg}$ of fluid removal, patients may develop postural hypotension. Risk of fall increases due to both lower muscle strength and hemodynamic conditions. However, in the present study, all participants performed the balance test before dialysis, because after dialysis, patients felt dry and weak, and were willing to stay to take the test. Had the balance test been performed after dialysis, the sensitivity of the test might have been improved, and the data might have better shown the benefits of the IMTC in reducing risk of fall. It is therefore recommended that in the future study, the balance test is performed after dialysis, if possible. No study has been reported on IMTC, although two studies $[19,50]$ have been published on interdialytic TC exercise, which is not for patients to perform while connected to the hemodialysis machine. In a pre- and post- pilot study, Mustata et al. [50] reported that 3 months of Wu-style-TC-based exercise training program, including 1 hour/session/week supervised in- hospital plus at least 2/ week home practice, improved QOL in patients on peritoneal dialysis. The limitations in Mustata's study included a small sample size $(n=6)$ and non-randomized control trial [50]. Ling et al. [19] reported that a home-based TC exercise program (30 minutes of TC 293 videotape program, 3-7/week) for 3 months improved functional mobility and muscle flexibility in ESRD patients. However, high attrition rate (54\%), relatively low adherence rate, and limited sample size $(n=33)$ in Ling's study explained the failure of the study to achieve statistically significant gains in many other outcome parameters, such as QOL and physical capacity [19]. TC has shown to benefit bone metabolism in non-dialysis population in terms of bone turnover biomarkers $[23,28,51,52]$. In the present study, relative to CON, we observed a decreased serum TRAP concentration in IMTC after 12 weeks $(p=0.056)$, indicating 
Citation: Phisitkul S, Chyu MC, Zhang Y, Brismee JM, Prabhakar S, et al. (2013) Intradialytic Modified Tai Chi Exercise among End-Stage Renal Disease Patients Undergoing Hemodialysis: An Exploratory Pilot Study. Altern Integ Med 2: 123. doi:10.4172/2327-5162.1000123

Page 6 of 7

that the IMTC exercise tended to suppress bone resorption in ESRD patients. This result appears to be consistent with Peppone et al. [52] that breast cancer survivors in the TC intervention group experienced a significant decrease in bone resorption, whereas those in the standard support therapy group did not. To investigate whether such suppressed resorption would translate into beneficial effects on bone metabolism in ESRD patients, a future study with a larger sample size is warranted. ESRD patients undergoing hemodialysis develop specific bone diseases including (i) adynamic bone disease related to low iPTH, and (ii) osteitis fibrosa cystic related to high iPTH. Extremely low or high iPTH level ( $<2$ or $>9$ times the upper normal limit) are undesirable and should be avoided in ESRD patients [48]. In the current study, no significant change in $\mathrm{PTH}$ was observed in IMTC relative to CON. Such result may be due to the following: (i) iPTH could also be regulated by other factors such as $1,25-\mathrm{OH}-\mathrm{VitD}$ dose and cinacalcet dose [53] that we did not control in this study, (ii) IMTC exercise had no weight bearing impact on ESRD patients compare to regular TC exercise, [23] and (iii) serum $\mathrm{Ca}$ and $\mathrm{P}$ levels were maintained within normal ranges in both CON and IMTC, which could help to stabilize the iPTH level. Serum 25-OH-VitD level in CON significantly decreased after 12 weeks, while that level was stable in IMTC. Serum 25-OH-VitD level reflects both endogenous synthesis from exposure to sunlight and intake from the diets including supplements [54]. The study participants in both CON and IMTC were prescribed the same dose of 25-OH-VitD supplement throughout the study. Among those participants who completed the study, we noticed that CON had a higher BMI than IMTC, although there was no statistical difference in BMI between these two groups at the baseline. Without sufficient information on the sun exposure time of study participants and with a relatively small sample size of the present study, it is not clear why CON experienced such a significant decrease in $25-\mathrm{OH}-\mathrm{VitD}$. In addition, it is unclear why $1,25-\mathrm{OH}-\mathrm{VitD}$ level in CON was higher than IMTC at baseline and 12 weeks. Although TC has shown to reduce urinary 8-OHdG in postmenopausal women, [26] we did not observe significant reduction in 8-OHdG in IMTC in the present study, possible due to the complexity of physiopathology in ESRD patients [16]. At 12 weeks, IMTC exercise benefited ESRD patients' QOL in terms of physical function, role physical and mental health (Table 4). The positive impacts of IMTC on physical function and role physical agree with previous report in patients with fibromyalgia [55]. The positive result of IMTC on mental health is consistent with our results in postmenopausal women [28].

The present study is limited in its small sample size. In order to better investigate IMTC's effects on risk of fall, the participants' number of falls before and after the intervention should be recorded in a study involving more ESRD patients at risk of fall. Although common in most studies involving exercise and ESRD patients, the relatively low compliance rate in IMTC may also render the insignificance of outcome parameters. Another limitation was related to the difficulty in controlling the different medications each participant received during the study as prescribed by his/her physician. We documented all changes in the medication prescribed by study patient's nephrologist, including phosphate binder and vitamin D. However, such changes were not considered in the data analysis. The amount of dietary calcium intake was not assessed, which could have potentially interfered with bone metabolism. Nevertheless, we did ask the study patients to maintain current dietary pattern throughout the study period. Their pre and post-intervention serum albumin report showed no changes in both groups.

\section{Conclusions}

This pilot study suggests that IMTC exercise is feasible and safe to ESRD patients undergoing hemodialysis. IMTC exercise improves QOL in terms of physical function, role physical, and mental health and maintains $25-\mathrm{OH}-\mathrm{VitD}$ level in such participants. The lack of significance in the results of balance, gait, and functional strength may be due to an already high performance in the participants at baseline. It is highly recommended future studies use a larger sample size, appropriate screening criteria for risk of fall aspects, and longer intervention period to further explore the efficacy of the IMTC exercise on bone health in ESRD patients.

\section{Acknowledgement}

We thank Texas Tech University Health Sciences Center for the funding support and the study participants and the Dialysis Center of Lubbock for participating in this study.

\section{Disclosure Statement}

All authors declare that no competing financial interest exists.

\section{References}

1. Wang Y, Jardine MJ (2011) Benefits of exercise training in patients receiving haemodialysis: a systematic review and meta-analysis. Br J Sports Med 45 1165-1166.

2. Jung TD, Park SH (2011) Intradialytic exercise programs for hemodialysis patients. Chonnam Med J 47: 61-65.

3. Johansen $\mathrm{KL}$ (2007) Exercise in the end-stage renal disease population. J Am Soc Nephrol 18: 1845-1854.

4. Konstantinidou E, Koukouvou G, Kouidi E, Deligiannis A, Tourkantonis A (2002) Exercise training in patients with end-stage renal disease on hemodialysis: comparison of three rehabilitation programs. J Rehabil Med 34: 40-45.

5. Moinuddin I, Leehey DJ (2008) A comparison of aerobic exercise and resistance training in patients with and without chronic kidney disease. Adv Chronic Kidney Dis 15: 83-96.

6. Painter $P(2005)$ Physical functioning in end-stage renal disease patients update 2005. Hemodial Int 9: 218-235.

7. Kouidi E, Grekas D, Deligiannis A, Tourkantonis A (2004) Outcomes of longterm exercise training in dialysis patients: comparison of two training programs. Clin Nephrol 61 Suppl 1: S31-38.

8. Painter P, Carlson L, Carey S, Paul SM, Myll J (2000) Low-functioning hemodialysis patients improve with exercise training. Am J Kidney Dis 36 $600-608$

9. Painter P, Carlson L, Carey S, Paul SM, Myll J (2000) Physical functioning and health-related quality-of-life changes with exercise training in hemodialysis patients. Am J Kidney Dis 35: 482-492.

10. Alem AM, Sherrard DJ, Gillen DL, Weiss NS, Beresford SA, et al. (2000) Increased risk of hip fracture among patients with end-stage renal disease. Kidney Int 58: 396-399.

11. Coco M, Rush $\mathrm{H}$ (2000) Increased incidence of hip fractures in dialysis patients with low serum parathyroid hormone. Am J Kidney Dis 36: 1115-1121.

12. Jamal SA, West SL, Miller PD (2012) Fracture risk assessment in patients with chronic kidney disease. Osteoporos Int 23: 1191-1198.

13. Mittalhenkle A, Gillen DL, Stehman-Breen CO (2004) Increased risk of mortality associated with hip fracture in the dialysis population. Am J Kidney Dis 44: 672-679.

14. Gillespie LD, Gillespie WJ, Robertson MC, Lamb SE, Cumming RG, et al. (2009) Interventions for preventing falls in elderly people. Cochrane Database Syst Rev 2: CD000340.

15. Schleicher MM, Wedam L, Wu G (2012) Review of Tai Chi as an effective exercise on falls prevention in elderly. Res Sports Med 20: 37-58

16. Low S, Ang LW, Goh KS, Chew SK (2009) A systematic review of the effectiveness of Tai Chi on fall reduction among the elderly. Arch Gerontol Geriatr 48: 325-331.

17. Lin MR, Hwang HF, Wang YW, Chang SH, Wolf SL (2006) Community-based 
Citation: Phisitkul S, Chyu MC, Zhang Y, Brismee JM, Prabhakar S, et al. (2013) Intradialytic Modified Tai Chi Exercise among End-Stage Renal Disease Patients Undergoing Hemodialysis: An Exploratory Pilot Study. Altern Integ Med 2: 123. doi:10.4172/2327-5162.1000123

tai chi and its effect on injurious falls, balance, gait, and fear of falling in older people. Phys Ther 86: 1189-1201.

18. Wong AM, Pei YC, Lan C, Huang SC, Lin YC, et al. (2009) Is Tai Chi Chuan effective in improving lower limb response time to prevent backward falls in the elderly? Age (Dordr) 31: 163-170.

19. Ling KW, Wong FS, Chan WK, Chan SY, Chan EP, et al. (2003) Effect of a home exercise program based on tai chi in patients with end-stage renal disease. Perit Dial Int 23 Suppl 2: S99-99S103.

20. Ott SM (2012) Bone disease in CKD. Curr Opin Nephrol Hypertens 21: 376381.

21. Coen G (2005) Adynamic bone disease: an update and overview. J Nephrol 18: $117-122$.

22. Exercise for Your Bone Health (2013).

23. Shen CL, Williams JS, Chyu MC, Paige RL, Stephens AL, et al. (2007) Comparison of the effects of Tai $\mathrm{Chi}$ and resistance training on bone metabolism in the elderly: a feasibility study. Am J Chin Med 35: 369-381.

24. Johnson-Davis KL, Fernelius C, Eliason NB, Wilson A, Beddhu S, et al (2011) Blood enzymes and oxidative stress in chronic kidney disease: a cross sectional study. Ann Clin Lab Sci 41: 331-339.

25. Roehrs M, Valentini J, Paniz C, Moro A, Charão M, et al. (2011) The relationships between exogenous and endogenous antioxidants with the lipid profile and oxidative damage in hemodialysis patients. BMC Nephrol 12: 59.

26. Shen CL, Chyu MC, Yeh JK, Zhang Y, Pence BC, et al. (2012) Effect of green tea and Tai Chi on bone health in postmenopausal osteopenic women: a 6-month randomized placebo-controlled trial. Osteoporos Int 23: 1541-1552.

27. Avramovic M, Stefanovic $V(2012)$ Health-related quality of life in different stages of renal failure. Artif Organs 36: 581-589.

28. Shen CL, Chyu MC, Pence BC, Yeh JK, Zhang Y, et al. (2010) Green tea polyphenols supplementation and Tai Chi exercise for postmenopausal osteopenic women: safety and quality of life report. BMC Complement Altern Med 10: 76.

29. Chyu MC, von Bergen V, Brismée JM, Zhang Y, Yeh JK, et al. (2011) Complementary and alternative exercises for management of osteoarthritis. Arthritis 2011: 364319.

30. Wang C, Schmid CH, Rones R, Kalish R, Yinh J, et al. (2010) A randomized trial of tai chi for fibromyalgia. N Engl J Med 363: 743-754.

31. Qiu P, Zhu W (2013) Tai Chi Illustrated. Human Kinetics.

32. Berg K (1989) Measuring balance in the elderly: preliminary development of an instrument. Physiotherapy Canada 41: 304-411.

33. Berg KO, Wood-Dauphinee SL, Williams JI, Maki B (1992) Measuring balance in the elderly: validation of an instrument. Can J Public Health 83 Suppl 2 S7-11.

34. Shumway-Cook A, Baldwin M, Polissar NL, Gruber W (1997) Predicting the probability for falls in community-dwelling older adults. Phys Ther 77: 812-819.

35. McConvey J, Bennett SE (2005) Reliability of the Dynamic Gait Index in individuals with multiple sclerosis. Arch Phys Med Rehabil 86: 130-133.

36. Chiu YP, Fritz SL, Light KE, Velozo CA (2006) Use of item response analysis to investigate measurement properties and clinical validity of data for the dynamic gait index. Phys Ther 86: 778-787.

37. Wolf SL, Barnhart HX, Kutner NG, McNeely E, Coogler C, et al. (1996) Reducing frailty and falls in older persons: an investigation of tai chi and computerized balance training. Atlanta FICSIT Group. Frailty and Injuries: Cooperative Studies of Intervention Techniques. J Am Geriatr Soc 44: 489497.

38. Xu L, Qian G, Tang L, Su J, Wang JS (2010) Genetic variations of hepatitis $B$ virus and serum aflatoxin-lysine adduct on high risk of hepatocellular carcinoma in Southern Guangxi, China. J Hepatol 53: 671-676.

39. Luo H, Tang L, Tang M, Billam M, Huang T, et al. (2006) Phase Ila chemoprevention trial of green tea polyphenols in high-risk individuals of liver cancer: modulation of urinary excretion of green tea polyphenols and 8-hydroxydeoxyguanosine. Carcinogenesis 27: 262-268
40. Wang JS, Busby WF Jr, Wogan GN (1995) Formation and persistence of DNA adducts in organs of CD-1 mice treated with a tumorigenic dose of fluoranthene. Carcinogenesis 16: 2609-2616.

41. Ware J Jr, Kosinski M, Keller SD (1996) A 12-Item Short-Form Health Survey: construction of scales and preliminary tests of reliability and validity. Med Care 34: $220-233$.

42. Pickard AS, Johnson JA, Penn A, Lau F, Noseworthy T (1999) Replicability of SF-36 summary scores by the SF-12 in stroke patients. Stroke 30: 1213-1217.

43. Cohen J (1992) A power primer. Psychol Bull 112: 155-159.

44. Ardenne M, Reitnauer PG (1975) Demonstration of tumor inhibiting properties of a strongly immunostimulating low-molecular weight substance. Comparative studies with ifosfamide on the immuno-labile DS carcinosarcoma. Stimulation of the autoimmune activity for approx. 20 days by BA 1 , a $\mathrm{N}$-(2-cyanoethylene)urea. Novel prophylactic possibilities. Arzneimittelforschung 25: 1369-1379.

45. Johansen KL, Chertow GM, Kutner NG, Dalrymple LS, Grimes BA, et al (2010) Low level of self-reported physical activity in ambulatory patients new to dialysis. Kidney Int 78: 1164-1170.

46. Cheema BS, Singh MA (2005) Exercise training in patients receiving maintenance hemodialysis: a systematic review of clinical trials. Am J Nephrol 25: 352-364.

47. Tentori F, Elder SJ, Thumma J, Pisoni RL, Bommer J, et al. (2010) Physica exercise among participants in the Dialysis Outcomes and Practice Patterns Study (DOPPS): correlates and associated outcomes. Nephrol Dial Transplant 25: 3050-3062.

48. KDIGO Clinical Practice Guideline for the Diagnosis, Evaluation, Prevention, and Treatment of Chronic Kidney Disease-Mineral and Bone Disorder (CKDMBD) Kidney Int (2009) 76: S50-S99.

49. Leung ES, Tsang WW (2008) Comparison of the kinetic characteristics of standing and sitting Tai Chi forms. Disabil Rehabil 30: 1891-1900.

50. Mustata S, Cooper L, Langrick N, Simon N, Jassal SV, et al. (2005) The effect of a Tai Chi exercise program on quality of life in patients on peritoneal dialysis: a pilot study. Perit Dial Int 25: 291-294.

51. Wayne PM, Kiel DP, Buring JE, Connors EM, Bonato P, et al. (2012) Impact of Tai Chi exercise on multiple fracture-related risk factors in post-menopausal osteopenic women: a pilot pragmatic, randomized trial. BMC Complement Altern Med 12: 7 .

52. Peppone LJ, Mustian KM, Janelsins MC, Palesh OG, Rosier RN, et al. (2010) Effects of a structured weight-bearing exercise program on bone metabolism among breast cancer survivors: a feasibility trial. Clin Breast Cancer 10: 224 229 .

53. Kumar R, Thompson JR (2011) The regulation of parathyroid hormone secretion and synthesis. J Am Soc Nephrol 22: 216-224.

54. Calvo MS, Whiting SJ, Barton CN (2005) Vitamin D intake: a global perspective of current status. J Nutr 135: 310-316.

55. Romero-Zurita A, Carbonell-Baeza A, Aparicio VA, Ruiz JR, Tercedor $P$, et al. (2012) Effectiveness of a tai-chi training and detraining on functional capacity, symptomatology and psychological outcomes in women with fibromyalgia. Evid Based Complement Alternat Med 2012: 614196. 